...a pathway of three genetic components controls agedependent cell death.

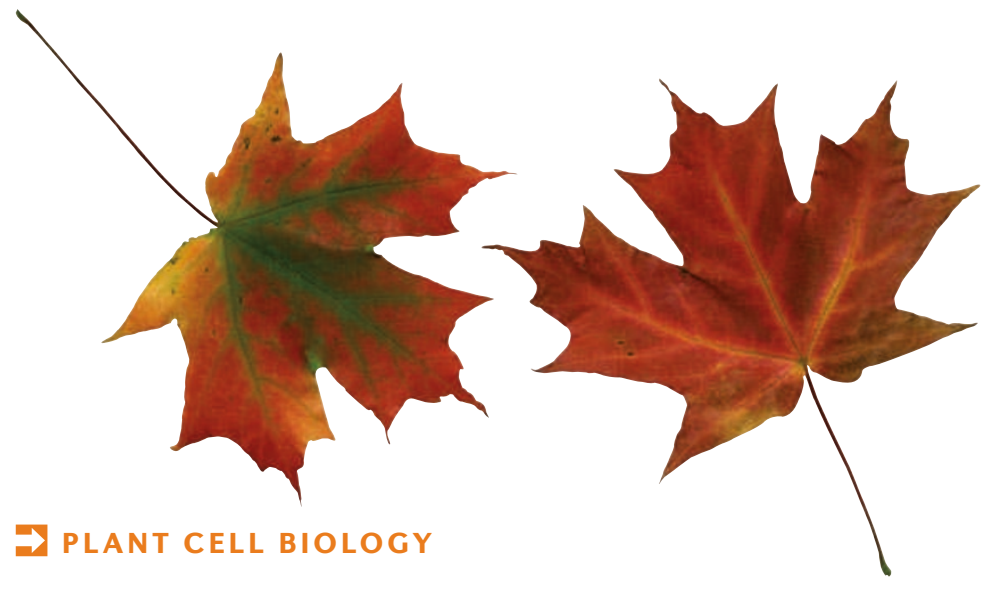

\title{
When autumn falls
}

Although the annual spectacle of leaves falling from deciduous trees is a familiar sight, the mechanisms behind the cell death events that precede autumnal leaf fall are poorly understood. Kim et al. now identify three components of a feed-forward loop - ORESARA 1 (ORE1), ETHYLENE INSENSITIVE 2 (EIN2) and the microRNA miR164 - that regulate age-dependent senescence in Arabidopsis thaliana leaves.

The authors studied the A. thaliana leaf senescence mutant ore1, which exhibits delayed ageingassociated cell death and delayed loss of chlorophyll, a marker of leaf senescence. They found that this phenotype is due to a 5 -base pair deletion in ORE1, which encodes a transcription factor. Wild-type ORE1 expression was increased in senescing leaves, suggesting a key role for ORE1 in this process.

miR164 binds and cleaves ORE1 mRNA. As plants age, the amount of miR164 in leaf cells decreases. Consequently, the authors tested whether miR164 might negatively regulate ORE1 $\mathrm{mRNA}$ and therefore age-dependent senescence. Plants that lack MIR164 have increased levels of ORE1 mRNA, whereas MIR164-overexpressing plants have decreased levels. In addition, the levels of an ore 1 mutant transcript that cannot bind miR164 are less affected by MIR164 overexpression. Therefore, miR164 binds and represses ORE1 mRNA and thereby delays age-dependent senescence.

But how is the temporal expression of MIR164 controlled such that ORE1 becomes less repressed as a leaf ages? By looking at other mutants in which cell death and leaf fall are delayed, the authors identified EIN2 as a further component of this genetic network. Because miR164 levels do not change with age in an ein2 mutant, wild-type EIN2 is thought to control the age-dependent reduction of miR164 levels. Furthermore, in older plants with the same ein 2 mutant, the level of ORE1 mRNA is substantially reduced. This suggests that EIN2 suppresses miR164mediated inhibition of ORE1 mRNA and also induces ORE1 expression.

These observations reveal that a pathway of three genetic components controls age-dependent cell death. Because EIN2 also has roles in cell growth control and stress response, it will be interesting to investigate whether this gene can link the genetic control of leaf cell senescence with seasonal environmental signals.

Simon Bishop

ORIGINAL RESEARCH PAPER Kim, J. H. et al.

Trifurcate feed-forward regulation of agedependent cell death involving miR164 in Arabidopsis. Science 323, 1053-1057 (2009) 